\title{
Painless Myocardial Ischemia Is Associated with Mortality in Patients with Chronic Kidney Disease
}

\author{
James B. Wetmore ${ }^{a, b}$ Mike Broce ${ }^{c}$ Amer Malas $^{d}$ Ammar Almehmi $^{\mathrm{e}}$ \\ ${ }^{a}$ Division of Nephrology and Hypertension and ${ }^{b}$ The Kidney Institute, University of Kansas Medical Center, \\ Kansas City, Kans., ' Center for Health Services and Outcomes Research, Charleston Area Medical Center Health \\ Education and Research Institute, ${ }^{d}$ St. Francis Medical Center, Charleston, W. Va., and ${ }^{~}$ Division of Nephrology, \\ University of Tennessee Health Science Center, Memphis, Tenn., USA
}

\section{Key Words}

Coronary artery disease $\cdot$ Myocardial ischemia $\cdot$ Chronic kidney disease $\cdot$ Mortality

\begin{abstract}
Background: Painless myocardial ischemia (PMI) is associated with poor outcomes in the general population. We hypothesized that the presence of PMI is inversely related to the level of kidney function and is associated with impaired survival in chronic kidney disease (CKD). Methods: A total of 356 patients who underwent percutaneous coronary intervention were assessed for $\mathrm{PMI}$, which was defined as the absence of chest pain in response to balloon dilation of the affected vessel. Cox proportional hazards analysis was used to calculate 10-year all-cause mortality. Results: There was an increase in PMI occurrence by strata of estimated glomerular filtration rate (eGFR), whereby PMI was present in only $20.6 \%$ of individuals with eGFR $\geq 90 \mathrm{ml} / \mathrm{min} / 1.73 \mathrm{~m}^{2}$, but was found in $50.0 \%$ of individuals with eGFR $<30 \mathrm{ml} / \mathrm{min} / 1.73 \mathrm{~m}^{2}(\mathrm{p}=$ 0.004 for trend). Classification of individuals as having either CKD or PMI showed significant differences in adjusted mortality between groups ( $p<0.001$ for trend), with individuals having both CKD and PMI demonstrating the highest 10year mortality. Compared to individuals with neither CKD
\end{abstract}

nor PMI, individuals with CKD and no PMI had a hazard ratio (HR) for mortality of 1.64 (95\% Cl: 1.03-2.63, $p=0.038$ ), while individuals with both PMI and CKD had an HR of 2.08 (1.30$3.33, p=0.002$ ). Conclusion: PMI is common in the CKD population, is inversely related to the level of eGFR, and confers a substantially increased risk in CKD. These findings may partially explain the high mortality traditionally attributed to cardiovascular disease in CKD patients.

Copyright $\odot 2013$ S. Karger AG, Base

\section{Introduction}

Cardiovascular disease (CVD) remains a major cause of morbidity and mortality in patients with chronic kidney disease (CKD), accounting for nearly half of all deaths $[1,2]$. A large proportion of these fatalities is attributed to sudden cardiac death [3], which is presumed to take the form of abrupt catastrophic ventricular arrhythmias. Patients with advanced CKD may be particularly susceptible to sudden cardiac death; for example $62 \%$ of cardiac deaths in chronic dialysis patients in the United States Renal Data System database are ascribed to arrhythmic mechanisms [4]. Arrhythmias appear to be relatively common in CKD patients when 24-hour ambulatory

\section{KARGER}

E-Mail karger@karger.com

www.karger.com/nec
(C) 2013 S. Karger AG, Basel

$1660-2110 / 12 / 1222-0009 \$ 38.00 / 0$
James B. Wetmore, MD, MS

University of Kansas Medical Center

MS 3002, 3901 Rainbow Blvd.

Kansas City, KS 66160 (USA)

E-Mail jwetmore@kumc.edu 
electrocardiography is employed, with some studies demonstrating that individuals with renal impairment not on dialysis have an increased prevalence of complex ventricular arrhythmias compared to individuals without renal disease [5]. Further, increased arrhythmic activity appears to be associated with a high prevalence of painless myocardial ischemia (PMI) in patients with low estimated glomerular filtration rate (eGFR), compared to individuals with intact kidney function [5], suggesting that PMI may be an underlying cause of arrhythmias and, as such, a major contributor to CVD mortality.

Several studies in the general population have shown that the presence of PMI may be more common than 'traditional' symptomatic angina and that PMI is associated with particularly poor outcomes [6,7]. Thus, understanding the importance of PMI and how it may contribute to CVD in at-risk populations, such as those with CKD, is imperative. PMI is well-documented in the dialysis population [8-11], and indeed the hemodialysis procedure itself it associated with regional wall motion abnormalities and alterations in myocardial perfusion [12]; however, very limited data are available on the prevalence of PMI and its prognostic implications in the CKD population [5]. In the present study, we sought to determine the frequency of PMI occurrence in nondialysis CKD patients undergoing coronary angioplasty of native coronary vessels, and to investigate the association between PMI, $\mathrm{CKD}$, and mortality during long-term follow-up. We hypothesized that the occurrence of provokable PMI is associated both with the stage of CKD and with particularly poor outcomes in patients with CKD.

\section{Subjects and Methods}

\section{Participants}

In this retrospective cohort study, we studied a total of 356 consecutive patients who underwent percutaneous coronary intervention (PCI) on native coronary vessels by a single operator between January 1999 and December 2001. We carefully selected our cohort using the following rationale. First, since our goal was to gain insights into the relationship between PMI and (nondialysis) CKD, we sought to study only patients who were not on dialysis. Second, we sought to study coronary disease which was both advanced but potentially remediable, so candidates had to undergo PCI. Finally, since previously bypassed patients differ in important ways from patients with de novo coronary disease $[13,14]$, we studied only individuals undergoing PCI on native vessels.

This registry was created and employed prospectively, with predefined domains of data collection applied to each enrollee. All patients underwent coronary artery stenting as clinically indicated; indications were pain thought to be likely of cardiac origin, shortness of breath, worsening heart failure, unstable angina, positive exercise stress test, or acute myocardial infarction. Patients with at least one available baseline creatinine value were included in the study. To enhance generalizability and reflect real-world clinical practice in the setting of PCI, the study criteria were deliberately broad; exclusion criteria were only dialysis dependence or intervention on nonnative vessels.

Data on demographics, cardiac risk factors, and laboratory results were obtained from the Charleston Area Medical Research Institution cardiac catheterization laboratory database and from other medical records. Operational definitions are as follows. Participants were considered to have previous CVD if he or she had prior myocardial infarction, PCI, coronary artery bypass graft surgery, peripheral vascular disease, or history of congestive heart failure not due to valvular disease. Diabetes was defined by the presence of a fasting blood glucose $\geq 126 \mathrm{mg} / \mathrm{dl}$ or a requirement for insulin or oral hypoglycemic agents; dyslipidemia by a total cholesterol level $>200$ $\mathrm{mg} / \mathrm{dl}$, low-density lipoprotein level $>100 \mathrm{mg} / \mathrm{dl}$, or use of an antihyperlipidemic agent; hypertension by systolic blood pressure (BP) $\geq 140 \mathrm{mmHg}$ and/or diastolic BP $\geq 90 \mathrm{~mm} \mathrm{Hg}$ at time of enrollment in the database, or use of antihypertensive medications; smoking by use of tobacco currently or within the past 6 months.

Stenotic lesions that reduced the coronary artery lumen by $50 \%$ or more on coronary angiogram were considered significant for this study. A standard conscious sedation protocol using midazolam (1-2 mg) and fentanyl (50-200 $\mu \mathrm{g})$ was utilized in all coronary interventions; at all times the patient remained sufficiently alert to answer questions and respond to instruction.

The study protocol was approved by the institutional review board of the Charleston Area Medical Center. At all times, the principles of the Declaration of Helsinki were adhered to.

\section{Estimation of GFR}

The abbreviated Modification of Diet in Renal Disease (MDRD) equation was used to estimate GFR [15]. The baseline creatinine on the day of admission was utilized. The National Kidney Foundation classification system was used to categorize individuals with CKD into respective stages according to their level of eGFR [16]. Participants with eGFR $<60 \mathrm{ml} / \mathrm{min} / 1.73 \mathrm{~m}^{2}$ were considered to have CKD.

\section{Definition of PMI}

Ischemia, which is traditionally classified as either painful or painless, can be induced when balloon angioplasty interrupts the blood supply to cardiac tissue perfused by that artery [17-19]. PMI can be identified either as a transient abnormality of the electrocardiogram documented on ambulatory monitor in the absence of angina or, in this case, as the absence of chest pain on coronary angioplasty during actual balloon inflation $[8,9,20,21]$. PMI was defined as the absence of chest pain (angina) or its equivalent (such as arm, throat, or jaw pain) in response to balloon dilatation in at least one affected vessel during PCI. A single operator consistently performed the intervention, while a nurse, who was not informed about the CKD status of the patient, queried the patient in standardized language about the presence of or absence of any pain during PCI.

\section{Statistical Analysis}

Data were presented as means \pm 1 SD for continuous variables and as proportions for categorical variables. The follow-up data were obtained by reviewing the electronic medical records and the catheterization laboratory database. Regarding demographics, in- 
Fig. 1. Classification of participants by presence of CKD and PMI.

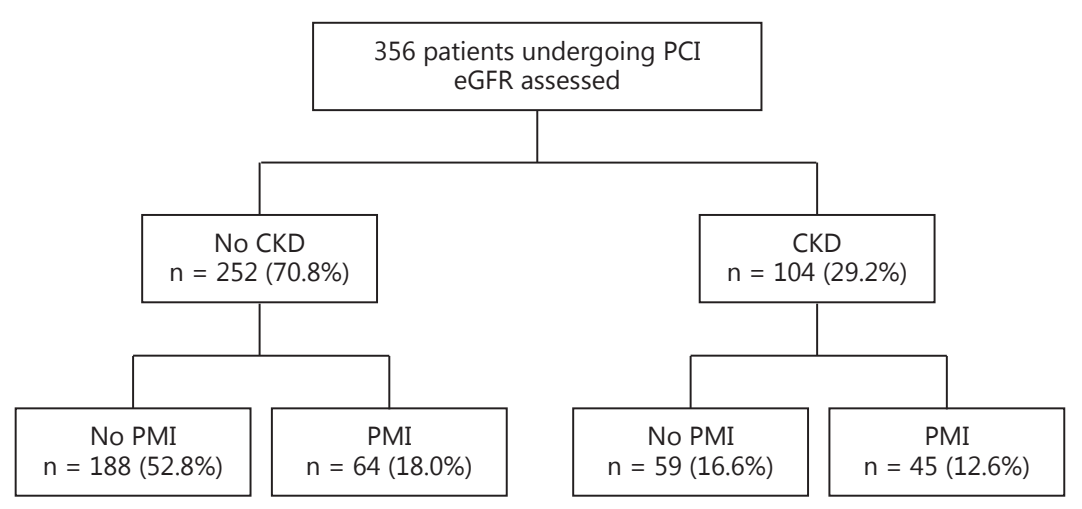

formation on race was not included in this analysis as non-Caucasians were $<2 \%$ of the study population, which is reflective of the racial distribution in West Virginia, USA. For the outcome analysis, participants were followed from the day of PCI until either death, the end of study (September 1, 2009), or loss to follow-up, at which point data were censored. The mortality data was ascertained by accessing the US Social Security Death Index in September 2009.

Differences between participants with and without PMI and CKD were analyzed using the $\chi^{2}$ test or Fisher's exact test for categorical data, the unpaired t test for continuous normally distributed data, and the Mann-Whitney test for non-normally distributed data. Because follow-up times were not uniform, Cox proportional hazards were employed for survival analysis. Cox proportional hazards modeling was used to create survival curves and detect resultant differences among the four groups of participants (i.e. the $2 \times 2$ matrix based on the presence or absence of PMI and $\mathrm{CKD}$ ). The following variables were used for adjustment in modeling: age, gender, specific cardiovascular risk factors (diabetes, hypertension, dyslipidemia, and smoking), chronic obstructive pulmonary disease, and cardiac medications (HMG-CoA reductase inhibitors or 'statins', $\beta$-blockers, and ACE inhibitors). Cox proportional hazards curves are presented as a figure, accompanied by the hazard ratios (HR) of the relevant variables in tabular form. Finally, to test the robustness of our results, a sensitivity analysis was then performed in which we increased the threshold for CKD by lowering the eGFR to $40 \mathrm{ml} / \mathrm{min} / 1.73 \mathrm{~m}^{2}$; this was done deliberately to increase the specificity of a diagnosis of presumed CKD. All analyses and calculations were conducted using SPSS statistical package version 15.0 (SPSS Inc., Chicago, Ill., USA). A p value of $<0.05$ was considered statistically significant.

\section{Results}

\section{Baseline Characteristics}

A total of 356 participants who underwent PCI demonstrated significant coronary artery disease. Figure 1 depicts the classification of participants by the presence or absence of CKD and PMI. Individuals with neither CKD nor PMI constituted the largest group (52.8\%), while the group with PMI and CKD was the smallest at $12.6 \%$. The distribution according to eGFR level was as follows: 16 (4.5\%) participants had eGFR $<30 \mathrm{ml} / \mathrm{min} / 1.73 \mathrm{~m}^{2}, 88$ (24.7\%) had eGFR $30-59 \mathrm{ml} / \mathrm{min} / 1.73 \mathrm{~m}^{2}, 152(42.7 \%)$ had eGFR $60-89 \mathrm{ml} / \mathrm{min} / 1.73 \mathrm{~m}^{2}$, and 100 (28.1\%) had eGFR $>90 \mathrm{ml} / \mathrm{min} / 1.73 \mathrm{~m}^{2}$.

Baseline characteristics are shown in table 1. Mean age was 64.6 years, and $64.0 \%$ were males. Patients with both CKD and PMI had higher absolute rates of diabetes and hypertension compared to the other groups. Individuals with both CKD and PMI, as expected, also had the highest absolute mean serum creatinine and blood urea nitrogen levels, as well as a lower eGFRs, resulting in significant trends across groups.

\section{Frequency of PMI}

PMI was more frequent in the CKD patients compared to those who had no CKD, 43.3 versus $25.4 \%$ ( $p=0.001$ ). As shown in figure 2, the frequency of PMI increased as the level of eGFR decreased; for example, in participants with eGFR $\geq 90.0 \mathrm{ml} / \mathrm{min} / 1.73 \mathrm{~m}^{2}$, PMI was present in $20.6 \%$, while in individuals with eGFR $<30.0 \mathrm{ml} / \mathrm{min} /$ $1.73 \mathrm{~m}^{2}$, the rate was fully $50.0 \%\left(\mathrm{p}_{\text {trend }}=0.004\right)$.

\section{Survival by Strata of CKD and PMI}

The maximum follow-up period was 10 years, while the mean follow-up time was $7.3 \pm 2.7$ years. A total of 143 participants died during the 10-year follow-up period; 213 participants survived. Adjusted Cox proportional hazards survival curves are shown in figure 3. Overall, mortality increased as disease burden increased: cumula- 
Table 1. Baseline characteristics stratified by CKD and PMI status

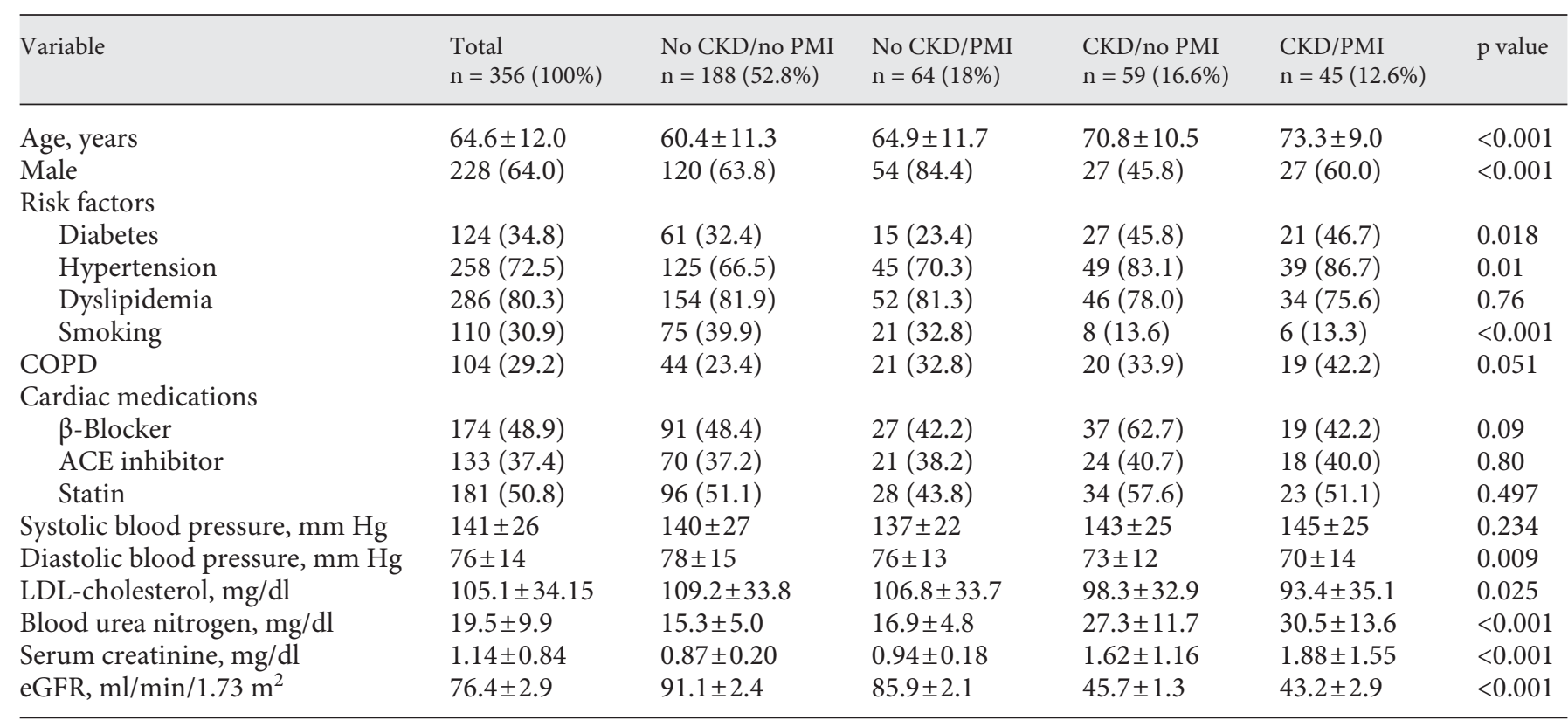

Values are means $\pm 1 \mathrm{SD}$ or $\mathrm{n}(\%)$. CKD defined as eGFR $<60 \mathrm{ml} / \mathrm{min} / 1.73 \mathrm{~m}^{2}$.

$\mathrm{COPD}=$ Chronic obstructive pulmonary disease; LDL = low-density lipoprotein; statin = HMG-CoA reductase inhibitor.

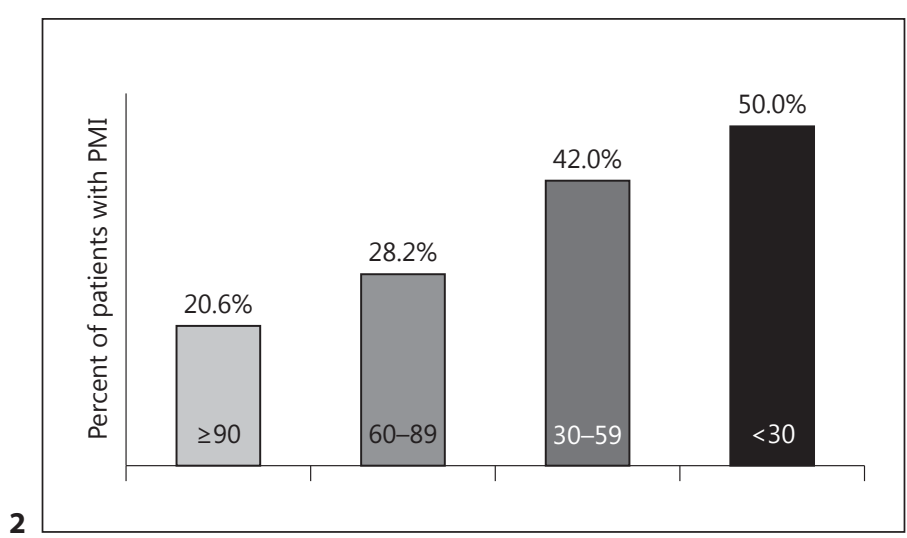

Fig. 2. Percent of participants with PMI by strata of eGFR (in ml/ $\left.\mathrm{min} / 1.73 \mathrm{~m}^{2}\right)$. Overall $\mathrm{p}=0.004\left(\chi^{2}\right.$ test for trend).

Fig. 3. Adjusted all-cause mortality in participants with and without PMI and CKD. Overall $\mathrm{p}<0.001$.

tive survival percentages for all-cause mortality at 10 years were $62.0 \%$ for individuals with neither CKD nor PMI, 45.2\% for individuals with PMI but intact renal function, $37.0 \%$ for individuals with CKD but no PMI, and $23.7 \%$ for individuals with both CKD and PMI. These

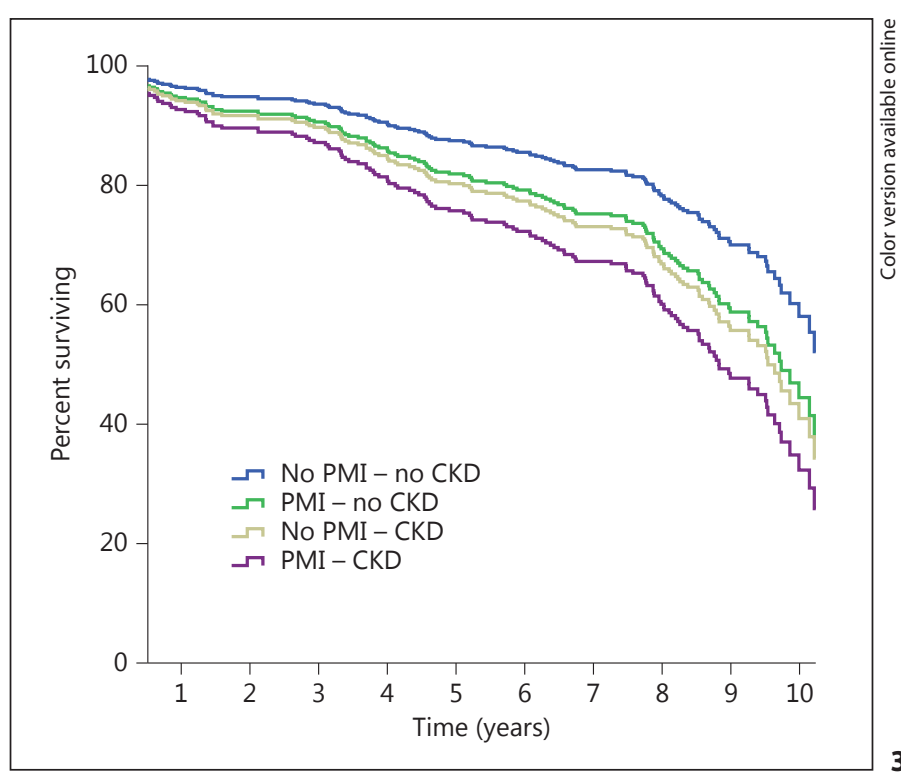

survival rates differed significantly across groups (overall $\mathrm{p}<0.001$ ).

Next, factors associated with mortality were modeled. After adjustment for differences in baseline characteristics and risk factors (as listed in table 1), age, hyperlipid- 
Table 2. Cox proportional HR for factors associated with all-cause mortality

\begin{tabular}{llcc}
\hline Variable & HR & $95 \%$ CI & p value \\
\hline Age, per year & 1.04 & $1.03-1.06$ & $<0.001$ \\
Dyslipidemia & 0.42 & $0.27-0.65$ & $<0.001$ \\
Diabetes & 1.27 & $0.90-1.79$ & 0.17 \\
COPD & 1.64 & $1.17-2.30$ & 0.004 \\
CKD-PMI interaction & & & \\
$\quad$ No CKD/no PMI & 1 & - & - \\
$\quad$ CKD/no PMI & 1.64 & $1.03-2.63$ & 0.038 \\
$\quad$ PMI/no CKD & 1.49 & $0.93-2.39$ & 0.098 \\
$\quad$ CKD and PMI & 2.08 & $1.30-3.33$ & 0.002 \\
\hline
\end{tabular}

emia, and COPD were significantly associated with mortality ( $\mathrm{p} \leq 0.004$ for each), while diabetes displayed a marginal effect $(\mathrm{p}=0.17$; table 2$)$. A significant interactive effect was found between CKD and PMI: compared to individuals with neither CKD nor PMI, CKD (in the absence of PMI) had an HR of 1.64 (95\% CI: 1.03-2.63, p = 0.038 ), while the simultaneous presence of both PMI and $\mathrm{CKD}$ increased the magnitude and significance of the HR to $2.08(1.30-3.33, \mathrm{p}=0.002)$.

To reduce the risk that CKD was being misclassified as acute kidney injury, we tested the robustness of our results by invoking a more stringent definition of CKD. CKD was redefined as having an $\mathrm{eGFR}<40 \mathrm{ml} / \mathrm{min} / 1.73 \mathrm{~m}^{2}$. In this analysis, we found a distinct effect of PMI, such that, compared to individuals with neither condition, PMI (in the absence of CKD) now demonstrated an HR of 1.58 (1.08$2.29, \mathrm{p}=0.017)$. The effect of simultaneous PMI and CKD was now somewhat stronger than before with an HR of 2.61 , although this was accompanied by somewhat wider confidence intervals $(1.18-5.79, \mathrm{p}=0.018)$.

Finally, to further explore our data, we examined the differences in the severity of coronary artery disease (i.e. the number of the affected vessels), the number of stents deployed, and the duration of balloon inflation as potential confounding factors. We found no significant differences in any of these among the four groups (table 3).

\section{Discussion}

In this study, we sought to determine whether the occurrence of PMI was greater in CKD patients than in individuals with intact renal function, and to examine the effect of PMI on mortality, independent of important traditional risk factors such as diabetes, in patients with
Table 3. Diseased coronary vessels and stents placed at the time of PCI

\begin{tabular}{lrll}
\hline eGFR group & $\mathrm{n}$ & $\begin{array}{l}\text { Number of } \\
\text { diseased vessels }\end{array}$ & $\begin{array}{l}\text { Number of } \\
\text { stents placed }\end{array}$ \\
\hline$<30$ & 16 & $1.4 \pm 0.5$ & $1.7 \pm 1.0$ \\
$30-59$ & 88 & $1.4 \pm 0.7$ & $1.9 \pm 1.1$ \\
$60-89$ & 152 & $1.4 \pm 0.7$ & $1.9 \pm 1.2$ \\
$\geq 90$ & 100 & $1.4 \pm 0.8$ & $2.2 \pm 1.2$ \\
\hline
\end{tabular}

Values represent means $\pm 1 \mathrm{SD}$.

${ }^{1} \mathrm{p}=0.93$ by ANOVA; ${ }^{2} \mathrm{p}=0.51$ by ANOVA.
CKD. We found a graded increase in the occurrence of PMI as the degree of renal impairment worsened, as well as a strong independent effect CKD on long-term survival in individuals with proven coronary artery disease. PMI appeared to potentiate the risk conferred by CKD in the primary analysis, and was frankly significant in our sensitivity analysis. Indeed, in the primary analysis, individuals with the combination of both CKD and PMI had a roughly 2.1-fold mortality risk over the ensuing decade compared to individuals with neither disorder, while the presence of CKD alone conferred a risk of roughly 1.6. These results suggest that the presence of PMI in CKD confers additional risk beyond that of CKD alone; therefore, PMI could be an important marker of increased allcause mortality in patients with CKD.

Our findings that PMI may contribute to mortality are broadly concordant with other studies. For example, Deedwania and Carbajal [7] evaluated the effect of PMI on mortality in 107 patients with stable angina, but with unknown renal function, and demonstrated a significant threefold increase in cardiac deaths in patients with PMI compared to those without PMI over a mean follow-up of 2 years. Other studies report more widely varying risk estimates, which may be due to differences in the patient populations and clinical scenarios examined (e.g. unstable angina as the presenting symptom) $[6,22]$. In another important observational analysis drawn from the Worchester Heart Attack Study, Sosnov et al. [23] analyzed the symptoms of myocardial infarction and their relationships to CKD. A finding of substantial interest was that patients with kidney disease were less likely to report chest pain compared to those without kidney disease. The authors concluded that kidney disease might change the perception of acute coronary disease. 
However, relatively little work has been done to rigorously examine the effects of both PMI and CKD on longterm survival. One such report is that of Aronow et al. [5], which demonstrated a significantly higher frequency of PMI in elderly patients with creatinine $>3.0 \mathrm{mg} / \mathrm{dl}$, compared to those with creatinine $<1.2 \mathrm{mg} / \mathrm{dl}$ (27 vs. $11 \%$, $\mathrm{p}=0.006$ ). While that important study appears to be the first to suggest that occurrence of PMI may be associated with the presence of CKD, it was limited by its small sample size and modest duration of follow-up. These limitations were remedied by the present investigation.

The mechanisms that underlie lack of ischemic symptoms in some patients, as well as the reasons why PMI is more common in individuals with CKD, are not well understood; several hypotheses, however, posit that these issues may be linked. One such hypothesis invokes left ventricular hypertrophy, which is common even in the early stages of CKD [24]. The development of left ventricular hypertrophy is associated with structural changes in the heart, resulting in myocyte-capillary mismatch which predisposes cardiomyocytes to hypoxia [25]. Left ventricular structural changes can also disrupt the precise patterning of cardiac innervation leading to altered neural activation and fatal ventricular arrhythmias [26, 27]. Another important mechanism proposes that a microinflammatory state, commonly seen in CKD patients, is responsible for PMI [28]. Indeed, accumulating evidence suggests that perception of angina is closely related to the microenvironmental balance between proinflammatory cytokines, such as IL- $1 \beta$, IL- 6 and TNF- $\alpha$, and anti-inflammatory cytokines such as IL-4 and IL-10 [29]. Regardless of the mechanism(s) responsible, it appears likely that PMI increases the ventricular arrhythmogenic activity and consequent cardiovascular death, particularly in vulnerable individuals such as those with $\operatorname{CKD}[5,30]$.

Of note, the risk conferred by diabetes was of only marginal statistical significance. This is not necessarily surprising given that conflicting evidence exists regarding the prevalence and clinical importance of PMI in diabetic patients. While some reports demonstrated a higher occurrence of PMI in diabetics compared to nondiabetics [31], others documented a higher or similar prevalence of symptomatic ischemia in diabetic and nondiabetic populations $[32,33]$. Importantly, Wackers et al. [34] reported that the lack of classical anginal symptoms in diabetics is not related to the traditional or the emerging cardiac risk factors, and that autonomic dysfunction appears to be a strong predictor of PMI [34, 35]. While autonomic dysfunction is not confined to diabetics (occurring, for example, in patients with vascular calcification), given the discrepancies in the literature about the association between PMI and diabetes, we were concerned that the latter could confound the effects of PMI on mortality, i.e. that adjustment for diabetes would attenuate the influence of PMI on the outcome. The fact that this was not observed in our analysis suggests the possibility that autonomic dysfunction may not fully explain the PMI observed in diabetes, or that autonomic dysfunction is also present in nondiabetic patients via other mechanisms. Much further work remains to be done in this area, particularly in individuals with CKD.

We acknowledge several important limitations to our study. Most fundamentally, our goals were circumscribed; as such, our findings may not be generalizable to the wider population. Specifically, our goal was to gain insights into the effect that CKD has on the occurrence of PMI in individuals with remediable native lesions. Since dialysis patients or patients with symptoms attributable to diseased bypass grafts differ from nondialysis patients with primary disease of the native vessels, our findings are probably limited to a restricted population of patients. Second, although data were prospectively collected, the analysis of the data was retrospective. Third, given the goals of the study, presence of a baseline creatinine was required for study inclusion, and thus patients without a baseline creatinine value were not solicited for participation; this represented $<5 \%$ of patients, and no data was collected on them. We believe this is only a modest limitation, however, since the vast majority of patients who undergo PCI have a preprocedural creatinine assessed. Fourth, recent creatinine values might not truly be reflective of CKD. That is, a patient could be experiencing acute kidney injury contemporaneously with the cardiac catheterization. This could potentially result in misclassification of acute kidney injury as $\mathrm{CKD}$, but in our experience major acute kidney injury prior to catheterization is fairly rare, and the effect of misclassification would have to be very large to completely undermine our results. We attempted to address this with our sensitivity analysis, in which we restricted the definition of CKD to individuals having an eGFR $<40 \mathrm{ml} / \mathrm{min} / 1.73 \mathrm{~m}^{2}$; reassuringly, the results were generally concordant with the findings of the primary analysis. It is also possible that residual confounding might still be present between potential risk factors and our outcome of interest; for example, we lack data on potential risk factors such as cardiac ejection fraction and albuminuria, both of which independently predict mortality while contributing to both renal and cardiac dysfunction $[15,36]$.

We also acknowledge that the clearance of opioid analgesics decreases as GFR falls [37]; while this could result 
in misclassification (i.e. CKD patients with symptomatic myocardial ischemia could be misclassified as painless), a single operator using an established protocol makes it less likely that variation in pain treatment practices substantially confounds our results. Further, patients more likely to have PMI might be expected to receive less overall pain medication, which could work to counterbalance the altered pharmacokinetics in CKD. A more rigorous analytical approach would universally fix the amount of pain medication administered, but such a study would pose considerable ethical challenges.

Finally, we also cannot rule out the possibility that the absence of overt chest pain may, paradoxically, be a surrogate for more severe coronary artery disease. Our goal, however, was to perform the first long-term study in a substantial number of patients in order to establish the potential risks of PMI and CKD and to generate hypotheses critical to informing future, more comprehensive, efforts. Electrocardiographic recordings during PCI, which could yield insights about the relationship between PMI and ST-segment changes, would be useful for future assessment [17]. Collectively, however, the limitations of this study are probably counterbalanced by the substantial number of patients studied, the long duration of follow-up, and the fact that a single operator recorded symptoms in rigorously systematic fashion utilizing the framework of prospectively designed registry.

It is important that our work be considered in its appropriate historical context. The last decade has witnessed dramatic changes in how PCI is used in clinical practice [38-41], which is reflected in evolving guidelines [42]. The increasing use of drug-eluting stents as well as refinement in the optimal use of antiplatelet therapy [38$40,42]$ mean that the long-term outcomes we report might differ were our study to be conducted today. Indeed, even the selection of appropriate PCI candidates may be changing, with an emerging belief that individuals with single vessel disease outside of the left anterior descending artery may not warrant PCI or even diagnostic catheterization [41]. However, the purpose of our study was to make foundational observations about how the perception of chest pain might vary by CKD status, a study which could only be performed in individuals who were, at the time, thought to be appropriate candidates for PCI with concomitant balloon occlusion. Comparable work should be performed in the present era to further inform the community on this important issue.

In conclusion, this is the first long-term study demonstrating that PMI is related to the degree of CKD and that the presence of PMI in CKD confers a risk greater than that of CKD alone. Although a full understanding of the molecular mechanisms contributing to PMI remains to be elucidated, PMI may be a key process in the disproportionate incidence of death attributed to arrhythmia in patients with CKD. Further studies are needed to assess the prevalence of PMI at different levels of eGFR in a prospective manner using screening modalities such as nuclear cardiac imaging, as well as to investigate the potential benefit of early intervention in patients with both PMI and CKD.

\section{Acknowledgments}

We are grateful to James Vacek, MD, for helpful discussions about the manuscript, and to Ms. Alesa Martin and Ms. Amanda Gellhaus for their technical assistance.

\section{Disclosure Statement}

The authors have no potential conflicts of interest to disclose. Support for this work was provided by NIH T32 training grant DK 071496 and National Kidney Foundation Renal Fellow Research Fellowship (A.A.).

\section{References}

1 Jaar BG, Coresh J, Plantinga LC, et al: Comparing the risk for death with peritoneal dialysis and hemodialysis in a national cohort of patients with chronic kidney disease. Ann Intern Med 2005;143:174-183.

-2 Vonesh EF, Snyder JJ, Foley RN, Collins AJ: The differential impact of risk factors on mortality in hemodialysis and peritoneal dialysis. Kidney Int 2004;66:2389-2401.

3 Pun PH, Smarz TR, Honeycutt EF, et al: Chronic kidney disease is associated with in- creased risk of sudden cardiac death among patients with coronary artery disease. Kidney Int 2009;76:652-658.

4 Herzog CA, Mangrum JM, Passman R: Sudden cardiac death and dialysis patients. Semin Dial 2008;21:300-307.

-5 Aronow WS, Ahn C, Mercando AD, Epstein S: Prevalence of coronary artery disease, complex ventricular arrhythmias, and silent myocardial ischemia and incidence of new coronary events in older persons with chronic renal insufficiency and with normal renal function. Am J Cardiol 2000;86:1142-1143, A9.

-6 Gottlieb SO, Weisfeldt ML, Ouyang P, Mellits ED, Gerstenblith G: Silent ischemia as a marker for early unfavorable outcomes in patients with unstable angina. N Engl J Med 1986;314:1214-1219.

7 Deedwania PC, Carbajal EV: Silent ischemia during daily life is an independent predictor of mortality in stable angina. Circulation 1990;81:748-756. 
${ }_{8}$ Mohi-ud-din K, Bali HK, Banerjee S, Sakhuja V, Jha V: Silent myocardial ischemia and high-grade ventricular arrhythmias in patients on maintenance hemodialysis. Ren Fail 2005;27:171-175.

-9 Narula AS, Jha V, Bali HK, Sakhuja V, Sapru RP: Cardiac arrhythmias and silent myocardial ischemia during hemodialysis. Ren Fail 2000;22:355-368

10 McIntyre CW, Burton JO, Selby NM, et al: Hemodialysis-induced cardiac dysfunction is associated with an acute reduction in global and segmental myocardial blood flow. Clin J Am Soc Nephrol 2008;3:19-26.

11 Dasselaar JJ, Slart RH, Knip M, et al: Haemodialysis is associated with a pronounced fall in myocardial perfusion. Nephrol Dial Transplant 2009;24:604-610.

-12 Assa S, Hummel YM, Voors AA, et al: Hemodialysis-induced regional left ventricular systolic dysfunction: prevalence, patient and dialysis treatment-related factors, and prognostic significance. Clin J Am Soc Nephrol 2012; 7:1615-1623.

13 Yamamuro M, Lytle BW, Sapp SK, et al: Risk factors and outcomes after coronary reoperation in 739 elderly patients. Ann Thorac Surg 2000;69:464-474.

14 Morrison DA, Sethi G, Sacks J, et al: Percutaneous coronary intervention versus coronary bypass graft surgery for patients with medically refractory myocardial ischemia and risk factors for adverse outcomes with bypass: the VA AWESOME multicenter registry: comparison with the randomized clinical trial. J Am Coll Cardiol 2002;39:266-273.

15 Levey AS, Bosch JP, Lewis JB, et al: A more accurate method to estimate glomerular filtration rate from serum creatinine: a new prediction equation. Modification of Diet in Renal Disease Study Group. Ann Intern Med 1999; 130:461-470.

$\$ 16 \mathrm{~K} / \mathrm{DOQI}$ clinical practice guidelines for chronic kidney disease: evaluation, classification, and stratification. Am J Kidney Dis 2002; 39:S1-S266.

17 Gutterman DD: Silent myocardial ischemia. Circ J 2009;73:785-797.

18 Cohn PF, Fox KM, Daly C: Silent myocardial ischemia. Circulation 2003;108:1263-1277.

19 Cleman M, Wohlgelernter D, Cabin H, et al: Myocardial dysfunction during percutaneous transluminal coronary angioplasty. Circulation 1990;81:IV14-IV19.
20 Wohlgelernter D, Jaffe CC, Cabin HS, Yeatman LA Jr, Cleman M: Silent ischemia during coronary occlusion produced by balloon inflation: relation to regional myocardial dysfunction. J Am Coll Cardiol 1987;10:491-498.

-21 Falcone C, Auguadro C, Sconocchia R, et al: Susceptibility to pain during coronary angioplasty: usefulness of pulpal test. J Am Coll Cardiol 1996;28:903-909.

22 Laukkanen JA, Kurl S, Lakka TA, et al: Exercise-induced silent myocardial ischemia and coronary morbidity and mortality in middleaged men. J Am Coll Cardiol 2001;38:72-79.

-23 Sosnov J, Lessard D, Goldberg RJ, Yarzebski J, Gore JM: Differential symptoms of acute myocardial infarction in patients with kidney disease: a community-wide perspective. Am J Kidney Dis 2006;47:378-384.

24 Levin A, Thompson CR, Ethier J, et al: Left ventricular mass index increase in early renal disease: impact of decline in hemoglobin. Am J Kidney Dis 1999;34:125-134.

25 Amann K, Breitbach M, Ritz E, Mall G: Myocyte/capillary mismatch in the heart of uremic patients. J Am Soc Nephrol 1998;9:10181022.

26 Ieda M, Fukuda K: New aspects for the treatment of cardiac diseases based on the diversity of functional controls on cardiac muscles: the regulatory mechanisms of cardiac innervation and their critical roles in cardiac performance. J Pharmacol Sci 2009;109:348-353.

27 Cao JM, Fishbein MC, Han JB, et al: Relationship between regional cardiac hyperinnervation and ventricular arrhythmia. Circulation 2000;101:1960-1969.

28 Carrero JJ, Stenvinkel P: Persistent inflammation as a catalyst for other risk factors in chronic kidney disease: a hypothesis proposal. Clin J Am Soc Nephrol 2009;4(Suppl 1):S49-S55.

29 Li JJ: Silent myocardial ischemia may be related to inflammatory response. Med $\mathrm{Hy}$ potheses 2004;62:252-256.

30 Parthenakis F, Kochiadakis G, Simantirakis E, et al: Incidence of ventricular arrhythmias during silent myocardial ischaemia in coronary artery disease. Int J Cardiol 1996;57:6167.

31 Ditchburn CJ, Hall JA, de Belder M, et al: Silent myocardial ischaemia in patients with proved coronary artery disease: a comparison of diabetic and non-diabetic patients. Postgrad Med J 2001;77:395-398.
32 Falcone C, Nespoli L, Geroldi D, et al: Silent myocardial ischemia in diabetic and nondiabetic patients with coronary artery disease. Int J Cardiol 2003;90:219-227.

-33 Caracciolo EA, Chaitman BR, Forman SA, et al: Diabetics with coronary disease have a prevalence of asymptomatic ischemia during exercise treadmill testing and ambulatory ischemia monitoring similar to that of nondiabetic patients. An ACIP database study. ACIP Investigators. Asymptomatic Cardiac Ischemia Pilot Investigators. Circulation 1996;93:2097-2105.

34 Wackers FJ, Young LH, Inzucchi SE, et al: Detection of silent myocardial ischemia in asymptomatic diabetic subjects: the DIAD study. Diabetes Care 2004;27:1954-1961.

- 35 Vinik AI, Maser RE, Mitchell BD, Freeman R: Diabetic autonomic neuropathy. Diabetes Care 2003;26:1553-1579.

-36 Kalantar-Zadeh K, Block G, Humphreys MH Kopple JD: Reverse epidemiology of cardiovascular risk factors in maintenance dialysis patients. Kidney Int 2003;63:793-808.

-37 Koehntop DE, Rodman JH: Fentanyl pharmacokinetics in patients undergoing renal transplantation. Pharmacotherapy 1997;17: 746-752.

38 Serruys PW: Fourth annual American College of Cardiology international lecture: a journey in the interventional field. J Am Coll Cardiol 2006;47:1754-1768.

39 Newsome LT, Kutcher MA, Royster RL: Coronary artery stents: part I. Evolution of percutaneous coronary intervention. Anesth Analg 2008; 107:552-569.

40 Newsome LT, Weller RS, Gerancher JC, Kutcher MA, Royster RL: Coronary artery stents: II. Perioperative considerations and management. Anesth Analg 2008;107:570590

41 Heyndrickx GR: Is functional assessment necessary in patients with stable angina? EuroIntervention 2010;6(Suppl G):G101-G106.

42 Blankenship JC, Moussa ID, Chambers CC, et al: Staging of multivessel percutaneous coronary interventions: an expert consensus statement from the Society for Cardiovascular Angiography and Interventions. Catheter Cardiovasc Interv 2012;79:1138-1152. 